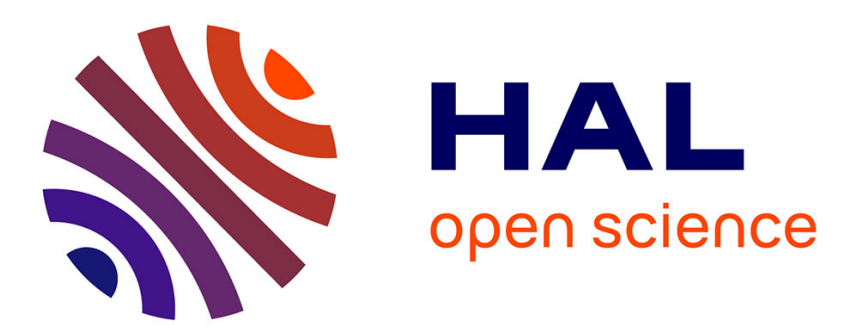

\title{
Contribution à l'étude d'une plante haploïde de pommier (Malus pumila Mill.). Etude descriptive et comparaison avec des clones de ploïdie différente. I. - Caractères végétatifs : entre- noeuds, feuilles et stomates \\ Yves Lespinasse, Dominique Noiton
}

\section{To cite this version:}

Yves Lespinasse, Dominique Noiton. Contribution à l'étude d'une plante haploïde de pommier (Malus pumila Mill.). Etude descriptive et comparaison avec des clones de ploïdie différente. I. - Caractères végétatifs: entre- noeuds, feuilles et stomates. Agronomie, 1986, 6 (7), pp.659-664. hal-00884922

\section{HAL Id: hal-00884922 \\ https://hal.science/hal-00884922}

Submitted on 1 Jan 1986

HAL is a multi-disciplinary open access archive for the deposit and dissemination of scientific research documents, whether they are published or not. The documents may come from teaching and research institutions in France or abroad, or from public or private research centers.
L'archive ouverte pluridisciplinaire HAL, est destinée au dépôt et à la diffusion de documents scientifiques de niveau recherche, publiés ou non, émanant des établissements d'enseignement et de recherche français ou étrangers, des laboratoires publics ou privés. 


\section{Contribution à l'étude d'une plante haploïde de pommier (Malus pumila Mill.). Etude descrip- tive et comparaison avec des clones de ploïdie différente. I. - Caractères végétatifs : entre- nœuds, feuilles et stomates}

Yves LESPINASSE \& Dominique NOITON $\left({ }^{1}\right)$

I.N.R.A., Station de Recherches d'Arboriculture fruitière, Beaucouzé, F 49000 Angers

Mots clés additionnels : Chromosomes, densité stomatique.

Contribution to the study of a haploid apple (Malus pumila). Descriptive study and comparison with clones of different levels of ploidy. I. - Vegetative characters : internodes, leaves and stomata.

A first haploid plant of apple has been studied and compared with related clones at diploid, triploid and tetraploid level. After a general description of the plant, the comparative study concerned internode length, leaf size, and stomatal size and density. The internodes of the haploid were distinctly shorter than those of the diploid and triploid. The very pointed leaves of the haploid had a leaf height/width ratio statistically different from those of the other clones; there was a regular decrease in this ratio, i.e. wider less pointed leaves, with increasing ploidy. Stomatal size gave the most clear-cut distinction between the clones compared.

Additional key words : Chromosomes, stomatal density.

\section{INTRODUCTION}

La recherche d'haploïdes de pommier par gynogenèse in vivo a débuté à l'INRA en 1975 ; l'intérêt de ces plantes pour l'étude cytogénétique et l'amélioration génétique de l'espèce a déjà été exposé (LESPINASSE \& GODICHEAU, 1980 ; LESPINASSE et al., 1982). La méthode utilisée repose sur la sélection de plantules d'origine maternelle repérées grâce à un

(1) Adresse actuelle: Department of Agronomy and Horticultural Science, University of Sydney, N.S.W. Australia 2006. marqueur anthocyanique. Parmi celles-ci, on pouvait espérer trouver des individus haploïdes résultant du fonctionnement parthénogénétique de l'une des cellules du sac embryonnaire. Effectivement, une première plante haploïde a été détectée dans la descendance de la variété «Topred Delicious » (LESPINASSE \& GODICHEAU, 1980), puis d'autres individus haploïdes ont été récemment décelés (LESPINASSE et al., 1983 ; CHEVREAU, 1984). Utilisant une autre méthode, l'androgenèse in vitro, des chercheurs chinois ont obtenu plusieurs haploïdes de pommier (WU, 1981 ; FEI \& XUE, 1981 ; WU et al., 1984 ; XUE \& NIU, 1984). Ce sont, à 
notre connaissance, les seules formes haploïdes de pommier obtenues dans le monde. Il faut toutefois signaler les 2 haploïdes décelés par EINSET (1945) après dénombrement chromosomique de descendants issus de variétés triploïdes en fécondation libre ; ces 2 individus n'ont pas survécu.

La plante haploïde issue de «Topred Delicious» a fait l'objet de plusieurs tentatives infructueuses de greffage à l'état herbacé sur jeunes plantes diploïdes issues de semis. Ce n'est qu'au stade ligneux que la greffe du clone haploïde a pu être réussie. La $1^{\text {re }}$ partie de cet article est consacrée, d'une part, à l'étude descriptive de la plante haploïde (aspect général de la croissance et morphologie des chromosomes) et, d'autre part, à l'étude comparative des dimensions des entre-nœuds, des feuilles et des stomates chez l'haploïde et 3 clones apparentés, diploïde, triploïde et tétraploïde. L'étude comparative portant sur la structure anatomique au point de greffe entre l'haplö̈de et les 2 clones apparentés diploïde et tétraploïde, fait l'objet de la $2^{\mathrm{e}}$ partie de l'article.

\section{MATÉRIEL ET MÉTHODES}

\section{A. Matériel}

L'origine des clones comparés, haploïde, diploïde, triploïde et tétraploïde est présentée dans le tableau 1. Leur ascendant commun est la variété «Delicious》; le clone diploïde «Topred Delicious » en dérive par mutation et le clone tétraploïde par doublement chromosomique. Le clone triplö̈de est un hybride issu d'un croisement entre la variété «Starkrimson » (voir tabl. 1) et une plante non apparentée sélectionnée pour sa résistance à la tavelure. Ces différents clones sont greffés sur des porte-greffe francs (plants de pommier diploïdes issus de semis) âgés d'un an. Malgré leur origine sexuée, ces porte-greffe présentent une homogénéité suffisante pour l'étude réalisée.

TABLEAU 1

Filiation des clones étudiés.

Filiation of the studied clones.

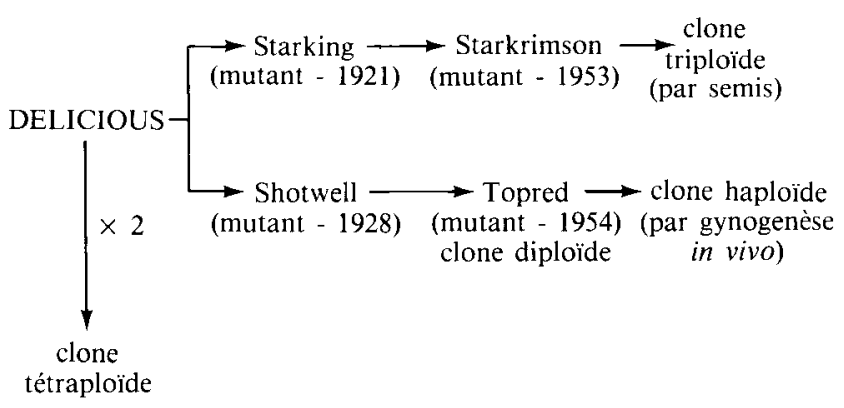

\section{B. Méthodes}

\section{Etude des chromosomes de l'haploïde}

Les chromosomes de l'hapleïde sont étudiés sur apex racinaires en utilisant une technique précédemment décrite (LESPINASSE \& SALESSES, 1973). Six pla- ques métaphasiques issues du même apex racinaire sont choisies ; les clichés obtenus sont examinés à la loupe binoculaire et les diverses mesures réalisées au micromètre. A partir de celles-ci, 2 paramètres sont calculés pour chaque chromosome : la longueur relative $\mathrm{L}_{\mathrm{r}}$ et l'indice centrométrique $\mathrm{I}_{\mathrm{c}}$ (DOSBA \& CAUDERON, 1973) :

$$
\mathrm{L}_{\mathrm{r}}=\frac{\mathrm{L}_{\mathrm{t}}}{\Sigma \mathrm{L}_{\mathrm{t}}} \times 17 \quad \mathrm{I}_{\mathrm{c}}=\frac{\mathrm{L}_{1}}{\mathrm{~L}_{\mathrm{t}}}
$$

$\mathrm{L}_{1}=$ longueur du bras long $\quad \mathrm{L}_{\mathrm{t}}=$ longueur totale $\Sigma \mathrm{L}_{\mathrm{t}}=$ somme des longueurs totales des 17 chromosomes.

2. Etude comparative: longueur des entre-nœuds, morphologie des feuilles, taille et densité des stomates

Les plants greffés sont mis en pots et élevés en serre dans des conditions de température et d'alimentation hydrique rigoureusement identiques. Toutes les mesures sont effectuées sur des rameaux provenant directement du greffon. Les comparaisons des moyennes sont réalisées à l'aide des tests de DUNCAN ou de NEWMAN \& KEULS (SOKAL \& ROHLF, 1969).

\section{a) Longueur des entre-nœuds}

Plusieurs rameaux sont mesurés à la fin de la période de croissance. Les 5 premiers (partie basale) et les 5 derniers entre-nœuds (partie apicale) ne sont pas pris en compte ; seule la partie médiane du rameau est donc considérée. Au total et pour chaque clone étudié, 64 entre-nœuds sont mesurés, mais ils ne correspondent pas toujours à un même nombre de rameaux.

\section{b) Morphologie des feuilles}

Il s'agit de caractériser chaque clone, à une date donnée, par les dimensions de l'ensemble des feuilles (jeunes, en croissance, et adultes) ; deux mesures sont effectuées : hauteur $(\mathrm{H})$ et largeur (L) du limbe. Les feuilles sont prélevées sur plusieurs rameaux et la relation entre hauteur et largeur du limbe est établie grâce à l'étude des régressions linéaires. Les rameaux du clone triploïde n'étaient plus en croissance à la date considérée ; nous ne disposons donc pour ce clone que de feuilles adultes.

\section{c) Taille et densité des stomates}

La taille et la densité des stomates sont étudiées sur des empreintes de la surface abaxiale des feuilles adultes. Pour chaque clone, 2 ou 3 feuilles sont prélevées dans la région médiane d'un rameau. L'empreinte est prise dans la partie centrale du limbe en pressant ce dernier sur un carré de collodion dont la surface a été ramollie par une goutte d'acétone pendant $1 \mathrm{mn}$ (SCHOCH \& SilvY, 1978).

La taille des stomates est estimée par la mesure de la longueur 1 des cellules de garde en utilisant un microscope équipé d'un oculaire à micromètre. Pour chaque clone, l'échantillon comporte 100 stomates. Le nombre de stomates par feuille peut s'exprimer soit 
par la densité stomatique (nombre de stomates par $\mathrm{mm}^{2}$ ou par $\mathrm{cm}^{2}$ ), soit par l'indice stomatique (pourcentage de stomates par rapport au nombre total de cellules épidermiques). Cet indice n'est pas utilisable chez le pommier car les cellules à contours très irréguliers sont difficiles à recenser; nous avons retenu la densité stomatique exprimée en nombre de stomates par $\mathrm{cm}^{2}$. Cette densité peut être transformée en distance moyenne entre chaque stomate (SLACK, 1974) ; soit ainsi la densité moyenne, $\mathrm{d}$, par $\mathrm{cm}^{2}$ :

Surface moyenne du limbe comportant un seul stomate, $\frac{\mathrm{l}}{\mathrm{d}} \mathrm{cm}^{2}$

Distance moyenne entre stomates,

$$
\mathrm{l}^{\prime}=\sqrt{\frac{\mathrm{l}}{\mathrm{d}}} \mathrm{cm}=\sqrt{\frac{1}{\mathrm{~d}} \times 10^{8}} \mu \mathrm{m}
$$

\section{RÉSULTATS}

\section{A. Etude descriptive de l'haploïde}

\section{Aspect général de la croissance}

Après 5 mois de croissance en serre, la jeune plante haploïde $(2 \mathrm{n}=\mathrm{x}=17)$ sur ses propres racines présente un phénotype en rosette aux entre-nœuds très tassés. Par la suite, la tige s'allonge et l'on obtient une plante ayant $70 \mathrm{~cm}$ de hauteur après 2 années de culture en serre. La ramification est de type basitone ; les rameaux, très grêles, portent des feuilles petites et allongées ; la croissance est lente. Cultivée sur ses pro pres racines, sa durée de vie a été de 4 ans ; la collecte répétée de greffons a sans doute hâté la mort de cet individu haploïde.

Après greffage sur porte-greffe "francs » et malgré de nombreuses difficultés de reprise du greffon (voir la $2^{\mathrm{e}}$ partie de cet article), la croissance est nettement améliorée au cours des 2 premières années (fig. 1). Par la suite, on note une nécrose des rameaux et une difficulté de survie. Il est vraisemblable que la surface foliaire très réduite du greffon conduit au dépérissement progressif du porte-greffe. De telles conditions naturelles de croissance laissent peu d'espoir d'observer un jour l'apparition de bourgeons floraux et, par conséquent, d'étudier le comportement méiotique de cet haploïde.

Les conditions de chambre phytotronique à température élevée $\left(32{ }^{\circ} \mathrm{C}-27^{\circ} \mathrm{C}\right)$ et éclairement de $16 \mathrm{~h}$ conduisent à une accélération de la croissance. Il est vraisemblable que l'environnement exceptionnel du phytotron puisse favoriser un développement végétatif suffisant et par la suite l'induction florale.

\section{Morphologie des chromosomes}

La longueur des chromosomes varie de 1,25 à $2,5 \mu \mathrm{m}$ (fig. 2). Le diagramme de distribution des 2 paramètres, $L_{r}$ et $I_{c}$, ne permet pas d'individualiser les 17 chromosomes du génome de base ; on peut toutefois tenter de les classer suivant leur longueur relative (tabl. 2). L'indice centrométrique varie de 0,5 à

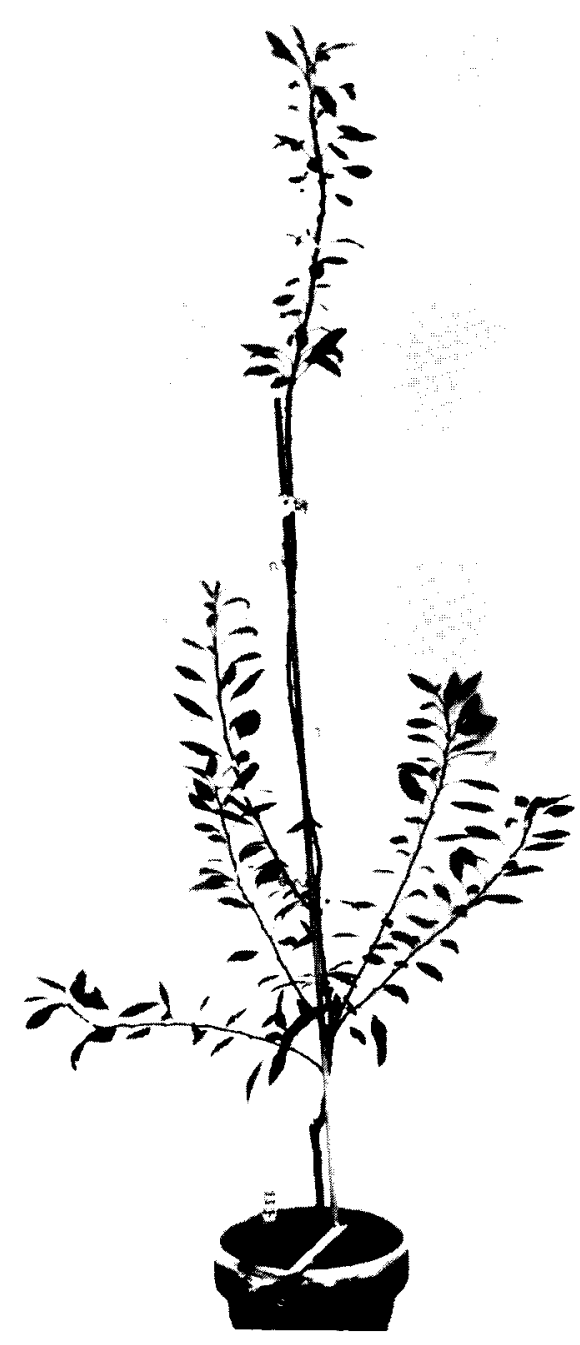

Figure 1

Plante haploïde greffée sur porte-greffe «franc» diploide après. 2 années de croissance végétative.

2 year old haploid plant after grafting on diploid apple seedling.

0,7 ; les chromosomes sont donc de type métacentrique à sub-métacentrique.

Le matériel étudié est vraisemblablement trop contracté pour aboutir à une distinction des différents chromosomes, la localisation du centromètre étant par ailleurs délicate. Des progrès pourraient être réalisés si l'on disposait d'une autre technique, par exemple celle qui a été appliquée au tabac et à divers Prunus après obtention de protoplastes par MOURAS et al. (1978).

\section{B. Etude comparative : longueur des entre-nouds, morphologie des feuilles, taille et densité des stomates}

\section{Longueur des entre-nouds}

La longueur moyenne des entre-nœuds exprimée en $\mathrm{mm}$ est de 11,2 pour le clone haploïde, 18,5 pour le clone tétraploïde, 28,5 et 30,5 respectivement pour le clone triploïde et le clone diploïde (tabl. 3 ).

Ces moyennes sont toutes significativement différentes (au seuil de probabilité du risque $\alpha=0,05$ ). 


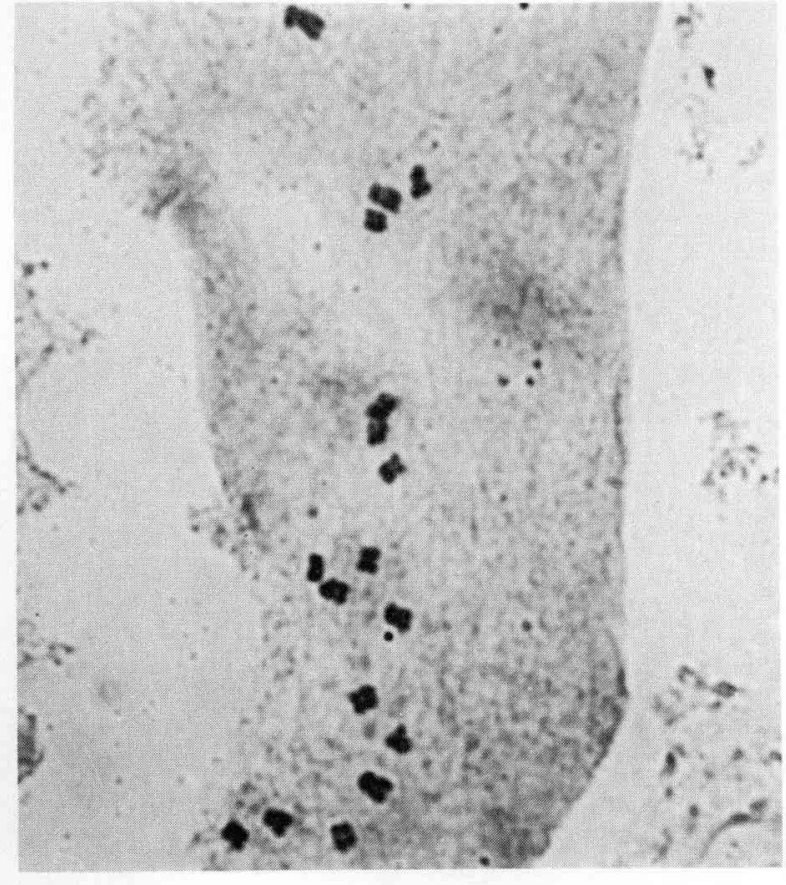

Figure 2

Cellule haploïde à 17 chromosomes provenant d'un apex racinaire. $(\times 1600)$.

Haploid cell with 17 chromosomes from a root tip. $(\times 1600)$.

TABLEAU 2

Répartition des 17 chromosomes du génome de base suivant leur longueur relative $L r$.

Distribution of the 17 chromosomes of the basic set according to their relative length $\mathrm{Lr}$.

\begin{tabular}{ccccc}
\hline \hline Classe Lr & $0,7-0,9$ & $0,9-1,0$ & $1,0-1,2$ & $1,2-1,4$ \\
\hline $\begin{array}{c}\text { Nombre de } \\
\text { chromosomes }\end{array}$ & 5 & 5 & 5 & 2 \\
\hline \hline
\end{tabular}

Les clones diploïde et triploïde présentent des longueurs d'entre-nœuds très voisines ; le clone haploïde se distingue nettement par des entre-nœuds très courts.
TABLEAU 3

Comparaison de la longueur des entre-nœuds chez les clones haploïde, diploïde, triploïde et tétraploïde.

Comparison between haploid, diploid, triploid and tetraploid clones for internode length.

\begin{tabular}{lc}
\hline & $\begin{array}{c}\text { Longueur moyenne X } \\
\text { (en mm) }\end{array}$ \\
\hline Haploïde & $11,2( \pm 0,5) \mathrm{a}^{*}$ \\
Diploïde & $30,5( \pm 1,0) \mathrm{b}$ \\
Triploïde & $28,5( \pm 1,0) \mathrm{c}$ \\
Tétraplö̈de & $18,5( \pm 0,9) \mathrm{d}$ \\
\hline
\end{tabular}

(entre parenthèses, intervalle de confiance au seuil de 95 p. 100). * Les valeurs suivies de lettres différentes sont significativement différentes au seuil de probabilité du risque $\alpha=0,05$.

\section{Morphologie des feuilles}

La relation qui existe entre les 2 mesures (hauteur $\mathrm{H}$ et largeur $\mathrm{L}$ du limbe) est étudiée par régression linéaire (tabl. 4). Les régressions et corrélations établies pour chaque niveau de ploïdie sont toutes significatives $(\alpha=0,05)$. Les coefficients de détermination calculés pour les clones haploïde, diploïde et tétraploïde montrent qu'une part importante de la variation de la hauteur est déterminée par la largeur du limbe (respectivement 86 p. $100 ; 85$ p. 100 ; 86 p. 100$)$.

Ces relations entre hauteur et largeur du limbe permettent de comparer les rapports moyens $\mathrm{H} / \mathrm{L}$ de chacun des clones (tabl. 5) ; seules les feuilles adultes ont été prises en considération.

Les résultats du test de comparaison des rapports $\mathrm{H} / \mathrm{L}$ montrent l'absence de différences significatives entre triploïde et tétraploïde (seuil $\alpha=0,05$ ) ; les feuilles haploïdes et diploïdes ont des rapports moyens $\mathrm{H} / \mathrm{L}$ significativement différents. Le rapport $\mathrm{H} / \mathrm{L}$ élevé du clone haplö̈de traduit bien la forme particulière, allongée et étroite, des feuilles de ce clone. Le rapport $\mathrm{H} / \mathrm{L}$, plus proche de $1 \mathrm{chez}$ les feuilles triploïdes et tétraploïdes, reflète la forme arrondie du limbe. Ces différences de morphologie des feuilles adultes sont illustrées par la figure 3 .

TABLEAU 4

Régression linéaire établie entre la hauteur $(H)$ et la largeur $(L)$ de la feuille pour les 4 clones étudiés. Linear regression between leaf height $(H)$ and width $(L)$ for the 4 clones studied.

\begin{tabular}{|c|c|c|c|c|}
\hline & $\begin{array}{l}\text { Equation } \\
\text { de la droite }\end{array}$ & $\begin{array}{l}\text { Signification } \\
\text { de la régression }\end{array}$ & $\begin{array}{c}\text { Coefficient } \\
\text { de corrélation }\end{array}$ & $\begin{array}{c}\text { Coefficient } \\
\text { de détermination }\end{array}$ \\
\hline $\begin{array}{l}\text { Haplö̈de } \\
(\mathrm{n}=101 \text { feuilles })\end{array}$ & $\mathrm{H}=2,04 \mathrm{~L}+4,58$ & $\begin{array}{l}\mathrm{t}=25,2 \\
\mathrm{t}_{0,05}=1,98\end{array}$ & $\mathrm{r}=0,93$ & 0,86 \\
\hline $\begin{array}{l}\text { Diploïde } \\
(\mathrm{n}=59 \text { feuilles })\end{array}$ & $\mathrm{H}=1,47 \mathrm{~L}+10,54$ & $\begin{array}{l}\mathrm{t}=17,8 \\
\mathrm{t}_{0,05}=2\end{array}$ & $r=0,92$ & 0,85 \\
\hline $\begin{array}{l}\text { Triploïde } \\
\text { (n = } 29 \text { feuilles) }\end{array}$ & $\mathrm{H}=1,58 \mathrm{~L}-2,89$ & $\begin{aligned} \mathrm{t} & =4,4 \\
\mathrm{t}_{0,05} & =2,04\end{aligned}$ & $r=0,65$ & 0,42 \\
\hline $\begin{array}{l}\text { Tétraploïde } \\
(\mathrm{n}=46 \text { feuilles })\end{array}$ & $\mathrm{H}=1,38 \mathrm{~L}+0,26$ & $\begin{array}{l}\mathrm{t}=40,7 \\
\mathrm{t}_{0,05}=2,02\end{array}$ & $\mathrm{r}=0,93$ & 0,86 \\
\hline
\end{tabular}


TABLEAU 5

Rapport $H / L$ (hauteur/largeur) des feuilles pour les 4 clones étudiés.

Ratio $H / L$ (height/width) in leaves for the 4 clones studied

\begin{tabular}{lccc}
\hline \hline & $\begin{array}{c}\text { Hauteur } \\
\text { moyenne } \\
\text { H (en mm) }\end{array}$ & $\begin{array}{c}\text { Largeur } \\
\text { moyenne } \\
\text { L (en mm) }\end{array}$ & Rapport H/L \\
\hline $\begin{array}{l}\text { Haplö̈de } \\
\text { (n=99 feuilles) }\end{array}$ & 36,3 & 15,5 & $2,34 \mathrm{a}^{*}$ \\
\hline $\begin{array}{l}\text { Diploïde } \\
\text { (n=49 feuilles) }\end{array}$ & 62,7 & 35,4 & $1,77 \mathrm{~b}$ \\
\hline $\begin{array}{l}\text { Triplö̈de } \\
\text { (n=29 feuilles) }\end{array}$ & 82,5 & 54,0 & $1,53 \mathrm{c}$ \\
\hline $\begin{array}{l}\text { Tétraploïde } \\
\text { (n = 38 feuilles) }\end{array}$ & 55,4 & 40,3 & $1,37 \mathrm{c}$ \\
\hline \hline
\end{tabular}

* Les valeurs suivies de lettres différentes sont significativement différentes au seuil de probabilité du risque $\alpha=0,05$.

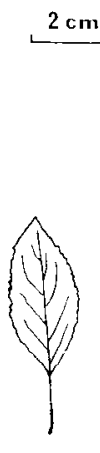

haploide

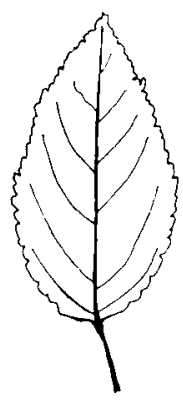

diploide

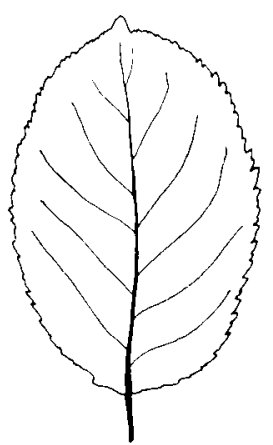

triploïde

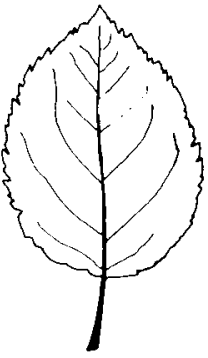

tètraploïde
Figure 3

Morphologie des feuilles adultes des divers clones étudiés.

Morphology of the mature leaves of the studied clones.

\section{Taille et densité des stomates (tabl. 6)}

En ce qui concerne la taille des stomates, la comparaison "multiple" des moyennes montre une différence significative au seuil $\alpha=0,05$ entre les 4 clones. Les densités stomatiques des clones haploïde et diploïde sont les plus élevées et la valeur la plus faible est notée chez le clone tétraploïde. Le test de compa-
TABLEAU 6

Taille et densité des stomates pour les 4 clones étudiés. Size and density of stomata for the 4 clones studied.

\begin{tabular}{llc}
\hline \hline & $\begin{array}{c}\text { Taille des stomates } \\
\text { en } \mu \mathrm{m}\end{array}$ & $\begin{array}{c}\text { Densité stomatique } \\
\text { par } \mathrm{cm}^{2}\end{array}$ \\
\hline Haploïde & $34,37( \pm 1,23) \mathrm{a}^{*}$ & $26973( \pm 1302) \mathrm{a}^{*}$ \\
Diploïde & $46,70( \pm 1,09) \mathrm{b}$ & $26816( \pm 1068) \mathrm{a}$ \\
Triploïde & $50,18( \pm 1,13) \mathrm{c}$ & $23604( \pm 1271) \mathrm{b}$ \\
Tétraploïde & $66,58( \pm 1,64) \mathrm{d}$ & $17207( \pm 1048) \mathrm{c}$ \\
\hline
\end{tabular}

(entre parenthèses, intervalle de confiance au seuil de 95 p. 100)

* Les valeurs suivies de lettres différentes sont significativement différentes au seuil de probabilité du risque $\alpha=0,05$.

raison des moyennes révèle une différence significative au seuil $\alpha=0,05$ entre les densités stomatiques des clones diploïde, triploïde et tétraploïde ; il en est de même pour le clone haploïde comparé au triploïde et au tétraploïde. Par contre, la différence entre haploïde et diploïde n'est pas significative.

\section{DISCUSSION}

Les caractères étudiés, longueur des entre-nœuds, morphologie des feuilles, taille et densité des stomates, permettent de bien différencier les plantes correspondant aux divers niveaux de ploïdie (tabl. 7). D'un point de vue statistique, les différences non significatives sont rares : les plantes de niveau triploïde et tétraploïde ne diffèrent pas pour le rapport $\mathrm{H} / \mathrm{L}$ des feuilles et celles de niveau haploïde et diploïde ne sont pas différentes quant à la densité stomatique. La longueur des stomates est le caractère le plus discriminant, la taille des cellules étant étroitement liée au degré de ploïdie.

Si l'on compare au niveau des stomates, longueur des cellules de garde et densité stomatique, on constate que les valeurs obtenues varient en sens inverse; ainsi, la plus faible densité stomatique s'observe chez le clone présentant les stomates les plus grands.

La densité stomatique convertie en distance moyenne entre stomates peut être comparée à la taille de ceux-ci par le calcul de l'indice $i=\frac{l^{\prime}}{l}$ (tabl. 7). On

TABLEAU 7

Récapitulation des résultats.

Summary of the results.

\begin{tabular}{|c|c|c|c|c|c|c|}
\hline & \multirow{2}{*}{$\begin{array}{c}\text { Entre-noeuds } \\
\begin{array}{c}\text { longueur } \\
\text { (en mm) }\end{array}\end{array}$} & \multirow{2}{*}{$\begin{array}{c}\text { Feuilles } \\
\text { rapport }\end{array}$} & \multicolumn{4}{|c|}{ Stomates } \\
\hline & & & $\begin{array}{r}\text { longueur } \\
(\mathrm{en} \mu \mathrm{m})\end{array}$ & $\begin{array}{c}\text { densité } \\
\left(\text { par } \mathrm{cm}^{2}\right)\end{array}$ & $\begin{array}{r}\text { distance } \\
(\mathrm{en} \mu \mathrm{m})\end{array}$ & indice $\mathrm{i}$ \\
\hline & $X$ & $\mathrm{H} / \mathrm{L}$ & 1 & d & $\mathrm{I}^{\prime}=\sqrt{\frac{1}{\mathrm{~d}} 10^{8}}$ & $\frac{1^{\prime}}{1}$ \\
\hline Haploïde & 11,2 & 2,34 & 34,4 & $26973\left(^{*}\right)$ & 60,9 & 1,77 \\
\hline Diploïde & 30,5 & 1,77 & 46,7 & $26816\left(^{*}\right)$ & 61,1 & 1,31 \\
\hline Triploïde & 28,5 & $1,53\left(^{*}\right)$ & 50,2 & 23604 & 65,1 & 1,30 \\
\hline Tétraploïde & 18,5 & $1,37\left(^{*}\right)$ & 66,6 & 17207 & 76,2 & 1,14 \\
\hline
\end{tabular}

(*) Différences non significatives au seuil $\alpha=0,05$. 
constate que les valeurs 1 et $1^{\prime}$ ne sont pas tout à fait directement proportionnelles sauf pour les clones diploïde et triploïde (valeurs de l'indice $\mathrm{i}$ respectivement de 1,31 et 1,30 ).

Si l'on considère l'ensemble des résultats, on remarque que les clones haploïde et tétraploïde se situent toujours aux extrêmes de l'échelle des moyennes comparées sauf dans le cas de la longueur des entrenœuds : le clone tétraploïde a une longueur d'entrenœuds supérieure à celle du clone haploïde mais nettement plus faible que celles des clones diplö̈de et triploïde. Cette distance inter-nodale traduit bien le faible développement du clone tétraploïde comparé à celui des clones diplö̈de et triploöde.

Avec l'augmentation du taux de ploïdie, les variations principales mises en évidence au cours de cette étude concernent l'augmentation de la longueur des stomates, la diminution de la densité stomatique et du rapport foliaire $\mathrm{H} / \mathrm{L}$ (hauteur sur largeur). L'évolution du rapport foliaire est certainement le résultat le plus original, du moins chez le pommier ; la figure 3 en est une bonne illustration. Une telle évolution de la morphologie des feuilles est un phénomène général, déjà mis en évidence chez d'autres végétaux (SANFORD, 1983). Une première observation d'une autre série apparentée haploïde, diploïde, triploïde et tétraploïde chez la variété de pommier «Golden Delicious » confirme cette évolution de la morphologie foliaire ; toutes les autres plantes haploïdes découvertes récemment sont caractérisées par des feuilles dont la morphologie est très proche de celle de la plante considérée dans cette étude. On remarque toutefois parmi les descendants issus de parents diploïdes, quelques individus à phénotype foliaire proche de la plante haploïde ; ils sont en fait diploïdes. Il était donc essentiel de conduire cette étude comparative entre des clones de filiation très proche.

Reçu le 25 mars 1985 Accepté le 3 avril 1986.

\section{RÉFÉRENCES BIBLIOGRAPHIQUES}

Chevreau E., 1984. Contribution à l'étude de la génétique du pommier : apport de l'analyse enzymatique. Thèse Doct. Ing., Orsay, $101 \mathrm{p}$.

Dosba F., Cauderon Y., 1973. Analyse statistique du caryotype d'Aegilops ventricosa Tausch. Ann. Amélior. Plantes, 23, 133-143.

Einset J., 1945. The spontaneous origin of polyploid apples. Proc. Am. Soc. Hortic. Sci., 46, 91-93.

Fei K. W., Xue G. R., 1981. Induction of haploid plantlets by anther culture in vitro in apple cv. " Delicious ». Sci. Agric. Sin., 4, 44 (résumé anglais).

Lespinasse Y., Godicheau M., 1980. Création et description d'une plante haploïde de pommier (Malus pumila Mill.). Ann. Amélior. Plantes, 30, 39-44.

Lespinasse Y., Salesses G., 1973. Application de techniques nouvelles à l'observation des chromosomes chez les genres Malus et Pyrus. Ann. Amélior. Plantes, 23, 381-386.

Lespinasse Y., Godicheau M., Noiton D., Duron M., 1982. L'haploïdie chez le pommier (Malus pumila Mill.). $2^{e}$ Colloque sur les Recherches Fruitières, Bordeaux, 19-27.

Lespinasse Y., Godicheau M., Duron M., 1983. Potential value and method of producing haploids in the apple tree, Malus pumila Mill. Acta Horticulturae, 131, 223-230.
Mouras A., Salesses G., Lutz A., 1978. Sur l'utilisation des protoplastes en cytologie : amélioration d'une méthode récente en vue de l'identification des chromosomes mitotiques des genres Nicotiana et Prunus. Caryologia, 31, 117-127.

Sanford J. C., 1983. Ploidy manipulations, p. 100-123. In : J. N. Moore \& J. Janick : « Methods in Fruit breeding ». Purdue University Press, West Lafayette, Indiana, 463 p.

Schoch P. G., Silvy A., 1978. Méthode simple de numération des stomates et des cellules de l'épiderme des végétaux. Ann. Amélior. Plantes, 28, 455-461.

Slack E. M., 1974. Studies of stomatal distribution on the leaves of four apple varieties. J. Hortic. Sci., 49, 95-103.

Sokal R. R., Rohlf F. J., 1969. Biometry. The principles and practise of statistics in biological research. Freeman and Co., San Francisco, $776 \mathrm{p}$.

Wu J., 1981. Obtaining haploid plantlets of crab apple from anther culture in vitro. Acta Hortic. Sinica, 8 (4), 36 (résumé anglais).

Wu J., Huang D., Han D., 1984. Haploid plant induction in apple (Malus pumila Mill.) and its cytological and histological study. Int. Symp. on Genetic Manipulation in Crops, Pékin, 22-26 Oct., 1984 (sous presse).

Xue G., Niu J., 1984. A study on the induction of apple pollen plants. Acta Hortic. Sinica, 11 (3), 164 (résumé anglais). 